\title{
Genome-Wide Investigation of the ZF-HD Gene Family in Two Varieties of Alfalfa (Medicago sativa L.) and Its Expression Pattern Under Alkaline Stress
}

Kai He

Northeast Agricultural University

Chunxin Li

Northeast Agricultural University

ZhenYue Zhang

Northeast Agricultural University

Lifeng Zhan

Northeast Agricultural University

Chunlong Cong

Northeast Agricultural University

DePeng Zhang

Northeast Agricultural University

Hua Cai ( $\triangle$ caihuaneau@gmail.com )

Northeast Agricultural University

\section{Research Article}

Keywords: Medicago sativa, ZF-HD gene family, Collinearity analysis, Alkaline stress

Posted Date: September 22nd, 2021

DOl: https://doi.org/10.21203/rs.3.rs-911266/v1

License: (c) (1) This work is licensed under a Creative Commons Attribution 4.0 International License.

Read Full License

Version of Record: A version of this preprint was published at BMC Genomics on February 21st, 2022. See the published version at https://doi.org/10.1186/s12864-022-08309-x. 


\section{Abstract}

\section{Background:}

Zinc finger homeodomain (ZHD) protein is a plant-specific transcription factor and a potential regulator of phosphoenolpyruvate carboxylase (PEPCase)-coding genes, and it also participates in plant growth regulation and abiotic stress responses. To study the function of MsZF-HD genes in the alkaline stress response, this paper assessed biological information and performed transcriptome analyses of the MsZF$H D$ gene family by using the genomes of two different varieties of alfalfa (XinJiangDa Ye and Zhongmu No. 1).

\section{Results:}

In total, 49 and $11 \mathrm{MsZF-HD}$ genes were identified in these varieties, respectively, including the alleles of XinJiangDa Ye. According to their phylogenetic relationships, the 60 MsZF-HD genes were divided into 5 ZHD subfamilies and 1 MIF subfamily. A total of $88.3 \%$ of MsZF-HD genes do not contain introns and are unevenly distributed among the 6 chromosomes of alfalfa. A collinearity analysis indicated that 26 genes of XinJiangDa Ye have no orthologous genes in Zhongmu No. 1, although these genes (such as ZHD-X12, ZHD-X3-2 and ZHD-X4-2) have homologous genes in Arabidopsis thaliana, Medicago truncatula and Glycine max. Through RNA-seq and qRT-PCR verification, it was found that MsZF-HD genes are downregulated to participate in the alkaline stress response.

\section{Conclusion:}

The results of this study may lay the foundation for the cloning and functional study of MsZF-HD genes and provide a theoretical basis for revealing the difference between XinJiangDa Ye and Zhongmu No. 1 at the genome level.

\section{Introduction}

Transcription factors (TFs) play a vital role in the growth and development of plants in response to abiotic stress by promoting the self-regulation and regulation of downstream target gene expression ${ }^{[1-2]}$. ZF-HD (zinc finger homeodomain, ZHD) is a plant-specific transcription factor that was first reported in the $\mathrm{C}_{4}$ plant Flaveria trinervia, and it is a potential regulatory factor of phosphoenolpyruvate carboxylase (PEPCase)-encoding genes ${ }^{[1]}$. The amino acid sequences of ZF-HD TFs have a conserved HD domain (homeodomain) that contains 60 amino acids that can be folded into a triple helix structure and specifically bind to the major groove of DNA ${ }^{[3-4]}$. In addition to the HD domain, ZF-HD also has a zinc finger structure (ZF domain) that is widely present in a variety of regulatory proteins and can specifically bind to DNA/RNA sequences and participate in protein interactions ${ }^{[5]}$. Interestingly, there is also a type of MIF (mini zinc finger) protein that is characterized by a very short sequence containing a central zinc finger domain ${ }^{[6]}$. Unlike the ZHD genes, MIFs are found only in seed plants and possibly originate from ZHDs by the loss of the homeodomain and subsequent divergence in seed plants; alternatively, it might 
be that ZHDs originated from MIFs by gaining the HD domain ${ }^{[7]}$. Thus, the ZHDs and MIFs should both belong to ZF-HDs, thereby dividing the ZF-HDs into two different groups. Currently, the ZF-HD family has been authenticated in Arabidopsis thaliana ${ }^{[2]}$, Vitis vinifera ${ }^{[8]}$, Glycine ${ }^{\left[x^{[9]}\right.}$, Solanum lycopersicum ${ }^{[10]}$, Fagopyrum tataricum $^{[11]}$, Triticum aestivum ${ }^{[12]}$, etc.

ZF-HD proteins are expressed predominantly or exclusively in floral tissue, indicating a likely regulatory role during floral development ${ }^{[2]}$. The ZF-HD class of homeodomain proteins may also be involved in the photosynthesis-related mesophyll-specific gene expression of phosphoenolpyruvate carboxylase in C4 species ${ }^{[2]}$ and in pathogen signaling and plant defense mechanisms ${ }^{[13]}$. Additionally, ZF-HD proteins were found to play various important roles in abiotic stress ${ }^{[14]}$. In $A$. thaliana, 17 ZF-HD family genes have been shown to have great significance in the development of flowers ${ }^{[1-2]}$. AtZHD1 is induced by drought, salinity and abscisic acid (ABA), and the overexpression of ZF-HD1 along with NAC genes confers tolerance to drought stress. It was also found that ZF-HD1 binds to the promoter of EARLY RESPONSE TO DEHYDRATION STRESS 1 (ERD1) ${ }^{[15]}$. Another report showed that AtMIF1 is overexpressed in Arabidopsis, suggesting that AtMIF1 may be involved in the regulation of plant development by multiple hormones. It is possible that AtMIF1 interacts with ZHD proteins via the ZF domain and its overexpression interferes with the normal functions of ZHD proteins ${ }^{[6]}$. Two soybean ZFHD proteins were confirmed to bind with the promoter of the gene encoding calmodulin isoform 4 (GmCaM4) and are highly expressed under pathogen inoculation ${ }^{[13]}$. In rice, 4 ZF-HD proteins were identified that bind to the promoter of OSDREB1B (DROUGHT RESPONSE ELEMENT BINDING 1B) and show differential transcriptional levels with OSDREB1B under different abiotic stress conditions, which is illustrative of the crosstalk between stress signaling pathways ${ }^{[16]}$. Additionally, OsZHD1, 2, 4, and 8 could form homo and heterodimers, and dimerization may play a prominent transactivation role in the regulation of the OSDREB1B response to abiotic stress.

Alfalfa (Medicago sativa L.) is the major forage protein source for livestock and has been planted in more than 80 countries around the world, with a planting area of more than 30 million hectares ${ }^{[17]}$. Due to the autotetraploidy and self-incompatibility of alfalfa, the lack of a reference genome makes artificial improvement very challenging. Although chromosome-level genome sequences based on Xin JiangDa $\mathrm{Ye}^{[18]}$ and Zhongmu No. ${ }^{[19]}$ have been published, the ZF-HD family has not been comprehensively analyzed in alfalfa. Hence, we used the genome of two different varieties of alfalfa to conduct a genomewide scan and bioinformatics analysis of the MsZF-HD genes. The analysis of this article may provide a valuable clue for further revealing the biological functions of $Z F-H D$ family genes and the cloning of MsZF-HD genes.

\section{Results}

\subsection{Identification of ZF-HD family genes in Medicago sativa L.}


A total of 49 MsZF-HD genes, including alleles, in XinJiangDa Ye and 11 genes in Zhongmu No. 1 were identified (only the longest splice variant was reserved at each genomic locus for further analysis). For the 60 putative MsZF-HD proteins, their molecular weight $(\mathrm{Mw})$ and isoelectric point $(\mathrm{pl})$ values were also determined by the Expasy online service (Supplementary Table 1). The protein sequences encoded by the M. sativa ZF-HD genes ranged in length from 75 amino acids for MsMIF-Z1 to 400 amino acids for MsZHD-X1-13, with an average of approximately 232 amino acids. The predicted Mw of the MsZF-HD proteins ranged from 8.14 to $43.36 \mathrm{kDa}$, and the theoretical pl values ranged from 5.05 to 9.07 . These results confirmed that the $60 \mathrm{MsZF-HD}$ proteins had large differences in sequence and protein characteristics.

\subsection{Phylogenetic analysis of M. sativa ZF-HD genes}

MEGA X software was used to construct a phylogenetic tree of $60 \mathrm{M}$. sativa and 17 Arabidopsis ZF-HDs

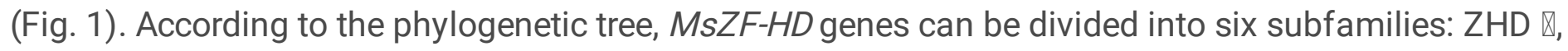
$Z H D \otimes, Z H D \otimes, Z H D \otimes, Z H D \otimes$ and MIF. The number of ZHD $\otimes$ subfamily proteins is the largest, with a total of $15 \mathrm{MsZHDs}$ and AtZHD5-7; while the number of ZHD $\otimes$ subfamily proteins is the least, with $5 \mathrm{MsZHDs}$ and $A t Z H D 1$. In addition, 5 Xin JiangDa Ye and 3 Zhongmu No.1 MIF proteins were assigned to the MIF subgroup and AtMIF1-3 were separately classified.

\subsection{Conserved motifs and gene structures}

To support the phylogenetic reconstruction, a motif analysis was performed by transferring the 60 MsZFHD protein and 17AtZF-HD sequences to the online MEME Web server (Fig. 2). Ten conserved motifs were identified in the MsZF-HD proteins, and the results were largely consistent with the phylogenetic analysis. Motif 2 and motif 3 were detected in most of the MsZF-HD proteins except for AtZHD-14, MsZHD-X2-3, MsZHD-X3-2, MsZHD-X2-13, MsZHD-X2-14 and 7 MIFs (including only motif 2 or motif 3). Each protein contains an average of 5 motifs, and the MIF subfamily contains only 1 or 2 . The motif distribution pattern of MsZF-HD proteins in each subfamily was basically the same, such that ZHD III subfamily members all contained motifs $2,3,6,7$ and 8 .

To identify the characteristics of the alfalfa ZF-HD gene family, the structure of these ZF-HD genes was analyzed (Fig. 2B). Most MsZF-HD genes do not contain introns, and only a few of them contain one intron, such as MsZHD-X1-3, MsZHD-X2-1, MsZHD-X2-5, MsZHD-X2-13, MsZHD-X3-6, MsZHD-X4-2 and MsZHD-Z2. The results revealed that members of the MIF and ZHD I subfamilies do not contain any introns.

\subsection{Chromosome distribution and gene duplication analysis}

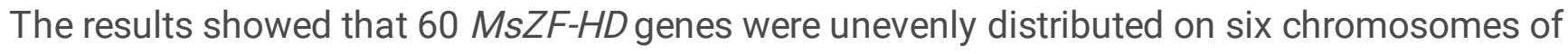
Xin JiangDa Ye and Zhongmu No. 1 while none were distributed on Chr2 and Chr4 (Fig. 3). The locations of MsZF-HD genes from the same subfamily were basically the same in XinJiangDa Ye and Zhongmu No. 1. For example, the chromosomes and relative positions of MsMIF-X1-1 and MsMIF-Z1 in the two 
varieties were fixed, indicating that the arrangement of MsZF-HD genes was highly conserved. When the query coverage and consistency of the candidate genes were $\geq 80$, they were considered repetitive genes. Chromosomal regions within the $200 \mathrm{~kb}$ range of two or more genes were defined as tandem replication events ${ }^{[20]}$. Therefore, the analysis of MsZF-HD gene duplication showed that only one tandem repeat gene (MsZHD-X1-11 and MsZHD-X1-12) was found on chromosome 8.1 in Xin JiangDa Ye and abundant genes were involved in fragment repeat events (Fig. 4).

To further infer the phylogenetic relationship between alfalfa and closely related dicotyledonous plants, we analyzed the collinear relationships between alfalfa and the other three plants (Fig. 5).

The results showed that $28 M s Z F-H D$ genes of Xin JiangDa Ye were collinear with $A$. thaliana genes, followed by G. $\max (17)$ and M. truncatula (15), and 7 MsZF-HD genes of Zhongmu No. 1 were collinear with $M$. truncatula and G. max genes, followed by $A$. thaliana (5). The collinear relationship between the MsZF-HD genes of Chr5 and that of the other three plants was significantly greater compared with that of the other chromosomes.

\subsection{Promoter region analysis of the MsZF-HD genes}

The identification of cis-regulatory elements in the promoter region can provide references for tissuespecific or stress-response expression patterns of genes. The 2.0-kb promoter region located upstream of the transcriptional start site (ATG) of each MsZF-HD gene was analyzed to determine their potential regulatory mechanisms by using online Plant CARE software. Several light response, hormone response and stress response elements were relatively highly abundant among these cis-elements. (Fig. 6). The ciselements of the MsZF-HD genes belonging to the same subfamily did not show the same pattern. Hormone-related cis-elements are predominantly represented by the MeJA (CGTCA-motif) and ABA (ABRE) response elements, which are endogenous growth regulators of higher plants and have physiological effects, such as inhibiting plant growth and germination, promoting senescence, and improving resistance (Fujita et al. 2013). These elements include 5 ABREs in MsZHD-X1-7 and 6 CGTCA motifs in MsZHD-X1-3, MsZHD-X1-5, and MsZHD-X2-13. Stress response elements can be divided into five categories: MBS, ARE, LTR, TC-rich repeats and WUN-motif. Among them, AREs (anaerobic response elements) were the most abundant, MsZHD-X4-5 and AtZHD9 all contained 6 AREs, and almost $80 \%$ of the upstream MsZF-HD genes contained ARE elements. In addition, Box-4, GT1 motifs, and G-boxes that respond to light were also prominent in the upstream MsZF-HD genes, especially Box-4. Ninety percent of the upstream MsZF-HD genes contained BOX-4 elements, and the number of these motifs was also the largest compared with the other motifs.

\subsection{Expression of MsZF-HD genes in response to abiotic treatment}

To investigate the molecular functions of the MsZF-HD genes in response to abiotic stress, the transcriptome data of Zhongmu No. 1 under different abiotic treatments $(250 \mathrm{mM} \mathrm{NaCl}, 10 \mathrm{M} \mathrm{ABA}$ and $400 \mathrm{mM}$ mannitol) were obtained from the $\mathrm{NCBI}$ and assembled into the genome of Zhongmu No.1 (Additional file 1: Table S2). The expression levels of these genes are presented in a heatmap (Fig. 7A). Of 
the 11 MsZF-HD genes in Zhongmu No. 1, the transcript levels (FPKM values) of three genes indicated that they were not expressed in any tissue while the remaining 8 genes were expressed at least once. Among them, most MsZF-HD genes were differentially expressed in all the examined treatments. Different genes showed different expression patterns; for example, MsZHD-Z5 showed a tendency of upregulation under all three treatments, while most genes (such as MsZHD-Z1, MsZHD-Z4, and MsMIFZ6) showed a low expression level. The expression of MsMIF-Z3 was upregulated under the ABA treatment, while the expression patterns of the mannitol and $\mathrm{NaCl}$ treatments were the opposite.

We also performed RNA-seq to detect the expression levels of the MsZF-HD gene under alkaline stress (Fig. 7B). After mapping those reads to the alfalfa genome, we determined the expression of 43 $M s Z F-H D$ genes that were expressed in at least one time period in the RNA-Seq data. All MsZF-HD genes decreased with time and could be clustered into three groups based on the expression profiles. Group I included genes with high expression levels, and members of subfamily I occupied the main position, followed by ZHD IV. Almost all genes in subfamily $\vee$ were not expressed, and the expression level of only two expressed genes (MsZHD-X1-11 and MsZHD-X2-13) belonging to group II was extremely low. ZHD II and III were almost all concentrated in group III, with low expression levels. To further confirm the RNAseq result of these MsZF-HD genes, qRT-PCR was performed for the MsZF-HD genes from different clusters (Fig. 7C). The expression patterns of most MsZF-HD genes in the qRT-PCR analysis were consistent with the RNA-Seq analysis, implying that our RNA-seq results are highly reliable. The expression level of most $Z F-H D$ genes was very low, and the $Z F-H D$ genes were downregulated after the alkaline treatment from $3 \mathrm{~h}$ to $48 \mathrm{~h}$.

\section{Discussion}

With the rapid development of high-throughput sequencing technologies, the ZF-HD gene family has been studied in many model plants, such as A. thaliana (17), G. max (36), T. aestivum (37), F. tataricum (20), V. vinifera (13), S. Iycopersicum (22), Gossypium hirsutum (35), Brassica napus (62), etc. In this study, 49 and 11 MsZF-HD genes were identified in XinJiangDa Ye and Zhongmu No. 1 by scanning the whole genome of two varieties of alfalfa, respectively. Among the four nonhomologous chromosome groups of Xin JiangDa Ye, the largest group was the second with 15 MsZF-HD genes, which was significantly larger than that of Zhongmu No. 1. Hence, the number of MsZF-HD genes was significantly less than that of other species. The number of MsZF-HD genes in XinJiangDa Ye was much higher than that of Zhongmu No. 1, which can lead to the formation of a flexible combinatorial system of transcription factors and may allow for subtle adjustments to many different environmental conditions because of the different binding to cis-elements and flexible dimerization.

To study the developmental and evolutionary relationship between M. sativa and Arabidopsis, a phylogenetic tree was constructed using all the ZF-HD protein sequences of Xin JiangDa Ye, Zhongmu No. 1 and Arabidopsis. The branches on these phylogenetic tree branches were similar to those in a previous Arabidopsis report, which means that most ZF-HD proteins have homology. Although there is a relatively close evolutionary distance between alfalfa and $M$. truncatula, the number of MsZF-HD 
gene homologous genes in $M$. truncatula is not greater than that in $G$. max. This finding may be related to the greater number of gene duplication events in G. max, which leads to more ZF-HD genes. Some MsZF$H D$ genes are related to at least two pairs of homologous genes, such as MsZHD-X3-2, MsZHD-X3-5 and $M s Z H D-Z 7$, which may play an important role in the evolution of the MsZF-HD family. In addition, there were 26 MsZF-HD genes in Xin JiangDa Ye that did not have orthologous genes in Zhongmu No. 1. However, these genes specific to Xin JiangDa Ye (such as MsZHD-X1-2, MsZHD-X1-3, and MsZHD$X 1-13)$ have homologous genes in the genomes of other dicotyledonous plants. It was proposed that increases or decreases in the genes on a single chromosome in the karyotype have a greater impact on the phenotype than changes in the whole genome ${ }^{[21]}$. Therefore, differences between the genomes of XinJiangDa Ye and Zhongmu No. 1 do exist, and these different genes are likely to be one of the reasons for the improved alkali tolerance of XinJiangDa Ye. There were a large number of collinearity relationships between nonhomologous chromosomes of alfalfa, and only a pair of tandem repeat genes were found in XinJiangDa Ye, indicating that the fragment repeats played an important role in the expansion of MsZF-HD genes. Tandem duplication of MsZF-HD genes was not observed in Zhongmu No. 1, which is consistent with the fewer MsZF-HD genes in Zhongmu No. 1, thus indicating that there were more active gene duplication events in Xin JiangDa Ye.

In general, TFs always harbor some important conserved domains and motifs for their regulatory function. Through the MEME suite, 10 motifs were identified in alfalfa. The distribution of conserved motifs in the same subfamily was basically consistent, and each conserved motif had its own unique function. Almost all ZF-HD proteins contained motif 2 and motif 3, while the MsZHD-X2-3, MsZHD-X3-2, MsZHD-X2-13, MsZHD-X2-14 and 6 MIF genes did not contain motif 2 or 3. A SMART online analysis showed that motif 2 and motif 3 were in the ZF-HD_dimer domain and indicated that they may be involved in the formation of homo or heterodimers and a zinc finger (Windhövel et al. 2001). These results indicate that the ZF-HD_dimer domain is highly conserved in ZF-HD proteins and plays an important role in binding DNA. There are three main mechanisms for changes in the structure of gene exons and introns (gain or loss, exonization or pseudoexonization, and insertion and deletion of exons or introns), which each lead to differences in gene structure ${ }^{[22]}$. When genes have no introns, they are not easy to connect and their functions are relatively conservative ${ }^{[23]}$. Almost all MsZF-HD genes have no introns, revealing that they play an important role in the growth and development of alfalfa. This result is consistent with a previously reported result showing that the ZF-HD genes have almost no introns ${ }^{[1]}$. Introns of MsZF-HD genes may be obtained by mutations during inheritance or evolution. The number of genes containing introns in Xin JiangDa Ye is greater than that of Zhongmu No. 1, and whether the appearance of introns in these genes affects the original functions is a question that needs further research.

ZF-HD genes are differentially expressed in different tissues of different species, indicating that they have an important role in the development of plant tissues ${ }^{[10]}$. ZF-HD TFs were first identified as a potential regulator of the PEPCase gene in the $\mathrm{C}_{4}$ plant Flaveria bidentis, and they are involved in a variety of biological processes, such as the response to abiotic stress and hormones, during plant 
development ${ }^{[2]}$. ZF-HD TFs have been shown to function in large complexes during the period in the early stages of flower development ${ }^{[24]}$, fruit development ${ }^{[11]}$, salt stress ${ }^{[25]}$ and drought stress ${ }^{[26]}$. However, the expression of $Z F-H D$ genes under alkaline stress has not been previously investigated.

Therefore, both XinJiangDa Ye and Zhongmu No. 1 were treated with alkaline stress and samples were taken at 5 different time periods to explore the expression of MsZF-HD genes in response to alkaline stress. Many MsZF-HD genes could not be detected after the alkaline treatment, which may indicate that these genes are expressed only in specific environmental conditions or tissues. The MsZF-HD genes from the same subfamily also have different expression patterns, indicating that these genes respond to alkali stress. All the MsZF-HD genes decreased over time under alkaline stress, which suggested that the response of these changes may be negatively regulated by abiotic stress. Most MsZF-HD genes expressed the highest levels in the early stage of the alkaline stress treatment and stabilized after 12 hours, reaching a very low level, thus indicating that the ZF-HD TFs respond to alkaline stress quickly and may be upstream of the signal transduction pathway. Constitutive overexpression of the AtMIF1 gene leads to significant developmental defects, including dwarfing, reduced apical dominance, altered flower morphology, reduced hypocotyl length, reduced root growth, and ectopic root hairs on hypocotyls and cotyledons ${ }^{[8]}$. Similar to the other ZD-HF genes, MsMIF also included a few genes that could respond to alkaline stress, although there were certain differences. The MIF subfamily genes of alfalfa have a low expression level, and only two expressed genes (MsMIF-X2-1 and MsMIF-X3-1) showed a trend of initially increasing and then decreasing.

\section{Conclusion}

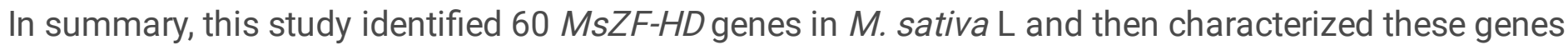
and explored their phylogenetic relationships. The expression analysis showed that in addition to drought, salt and ABA stress, most MsZF-HDs also respond to alkaline stress.

\section{Materials And Methods}

\subsection{Identification and characterization of the ZF-HD family in alfalfa.}

The genome and annotation information of alfalfa (Xin JiangDa Ye and Zhongmu No. 1) were downloaded from the figshare data repository (https://figshare.com). The $A$. thaliana genome and annotation information were downloaded from the TAIR database (https://www.arabidopsis.org/), and the 17 AtZF-HD protein sequences were downloaded from TFDB (http://planttfdb.gao-lab.org/, V5.0). The MsZF-HD genes were searched using 2 methods. First, the published AtZF-HD protein sequences were used as probes for a BLAST search against the proteome of alfalfa to obtain candidate ZF-HD family genes. Second, the hidden Markov model (HMM) profiles of the ZF-HD_dimer domain (PF04770) were downloaded from the Pfam database (http://pfam.xfam.org/) to search for the ZFHD_dimer domain from the alfalfa proteome using an e-value cut off of 1.0. Duplicate genes obtained by the two methods were removed to obtain the final MsZF-HD genes. The NCBI Batch CD-Search (https://www.ncbi.nlm.nih.gov) was utilized to analyze the domains of candidate ZF-HD proteins to 
ascertain the candidate genes for subsequent analysis. Finally, the nucleotide and deduced amino acid sequences of $60 \mathrm{MsZF-HD}$ genes were confirmed for further analysis. The amino acid sequence lengths, molecular weights (MW) and pl values of all predicted MsZF-HDs were then determined by ExPASy (http://web. expasy.org/compute_pi/). All related information of MsZF-HD genes are listed in Table 1.

\subsection{Phylogenetic analysis of ZF-HD proteins}

To study the phylogenetic relationships of the different ZF-HDs, the full-length amino acid sequences of ZF-HD members of alfalfa and Arabidopsis were used to construct phylogenetic trees by the maximum likelihood (ML) method with 1000 bootstrap replicates through MEGA X software. The parameters were set as follows: protein model: $\mathrm{JTT}+\mathrm{G}+\mathrm{l}$; gap/missing date treatment: partial deletion; and site coverage cut off: $95 \%$.

\subsection{Conserved motif, gene structure, and cis-element analysis of the ZF-HD gene family in alfalfa}

Conserved motifs and domains of ZF-HD genes were predicted by MEME (https://memesuite.org/meme/tools/meme). The optimized parameters of MEME were as follows: the optimum width of each motif ranged from 6 to 50, and the maximum number of motifs to find was 10. In addition, TBtools ${ }^{[27]}$ was used to display the gene structure and identify the exon/intron boundaries. The Plant CARE database (http://bioinformatics.psb.ugent.be/webtools/plantcare/html/) was used to predict and analyze the cis-elements of 2000 bp upstream sequences of each alfalfa $Z F-H D$ gene.

\subsection{Chromosomal localization and collinearity analysis}

To analyze the distribution of MsZF-HD genes in alfalfa chromosomes, reference information for the alfalfa genome was obtained. The chromosomal distribution and synteny were analyzed using TBtools. The proteomes of M. sativa L., A. thaliana, Medicago truncatula, and G. max were blasted to find duplicated genes between different species. Multiple collinear scanning toolkits (MCScanX) were used to analyze the replication events of the MsZF-HD genes. The syntenic relationship between the MsZF-HD genes and ZF-HD genes from selected plants was determined using Dual Systeny Plotter software (https://github.com/CJ-Chen/TBtools).

\subsection{Naming of ZF-HD genes}

We considered a consistent naming pattern for all MsZF-HD genes, with the phylogenetic relationships as well as their subgenome location in XinJiangDa Ye (1, 2, 3 or 4$)$ taken into account. Each gene name starts with an abbreviation for the species, e.g., Ms for M. sativa L., followed by the name of the subfamily, e.g., 'ZHD' for 'zinc finger motif-associated HD' and 'MIF' for 'mini zinc finger'. In addition, Z and $X$ indicate which variety they represent. The MsZF-HD genes belonging to XinJiangDa Ye but on different homologous chromosomes were consecutively numbered and separated by a dash. Hence, the 
name of the gene with the ID MS.gene062215 is MsZHD-X1-1, indicating that it is a ZHD subfamily gene and the first homologous chromosome and first gene of XinJiangDa Ye.

\subsection{Plant material and stress treatment}

M. sativa (Xin JiangDa Ye and Zhongmu No. 1) was employed for the alkaline treatment experiments. Uniform seedlings were transplanted into plastic cylindrical pots $(10.5 \mathrm{~cm}$ diameter $\times 9.5 \mathrm{~cm}$ high with a $5 \mathrm{~mm}$ diameter hole at the bottom, three plants per pot) containing vermiculite and perlite (1:1) under $16 / 8 \mathrm{~h}$ light/dark conditions, $30^{\circ} \mathrm{C} / 25^{\circ} \mathrm{C}$ day/night temperatures and $55 \%$ relative humidity. The pots were placed in rectangular plastic trays $(55 \mathrm{~cm} \times 75 \mathrm{~cm}, 24$ pots per tray). During plant growth, $1 / 5$ Hoagland nutrient solution was applied to the plants once every 2 days. Two-month-old seedlings were utilized for the alkaline treatment. For alkaline stress, plants were treated with $250 \mathrm{mmol} / \mathrm{L}$ $\mathrm{NaHCO}_{3}(\mathrm{pH}=8.5)$, and leaves of the seedlings were harvested at $0,3,6,24$, and $48 \mathrm{~h}$ after treatment. Immediately, the collected samples were frozen and stored at $-80{ }^{\circ} \mathrm{C}$ until use. For all the above samples, three biological replicates were employed for each sample.

\subsection{Medicago sativa RNA-seq data analysis}

M. sativa transcriptome data under different abiotic stresses were downloaded from the NCBI SRA database (http://www.ncbi.nlm.nih.gov, accession numbers SRX4079528-SRX4079572). Different time points of three abiotic stresses, including $250 \mathrm{mmol} / \mathrm{L} \mathrm{NaCl}, 10 \mathrm{~mol} / \mathrm{L} \mathrm{ABA}$, and $400 \mathrm{mmol} / \mathrm{L}$ mannitol, were analyzed. The transcript abundance is expressed based on the fragments per kilobase of exon model per million mapped reads (FPKM) values. Heat maps for MsZF-HD genes that have positive FPKM values in at least one or more of the samples were generated, and the values are shown with log2 $(1+$ RPKM). We also performed RNA-seq to detect the expression levels of ZF-HD TF genes under alkaline stress. Clean reads from thirty samples were mapped to the $M$. sativa genome sequencing using SamTool ${ }^{[28]}$. TopHat and Cufflinks were used to analyze the FPKM results ${ }^{[29]}$. The FPKM values for ZFHD genes were utilized to generate the heatmap and k-means clustering using R (software) ${ }^{[30]}$.

\subsection{RNA extraction and qRT-PCR verification}

Total RNA was extracted from each sample using the Plant RNAprep Pure Kit (Kangwei, Beijing China). First-strand cDNA was synthesized from $1 \mu \mathrm{g}$ of total RNA using a HiScript II Q Select reverse transcriptase kit (Vazyme Biotech, Nanjing, China) according to the manufacturer's instructions. Genespecific primers were designed using Primer Premier 5 software. In each reaction, the GAPDH gene (accession: XM_003601780.1) was used as an internal reference gene. The relative gene expression levels were calculated according to the $2^{-\Delta \Delta C(t)}$ method. SPSS 2.5 and Excel software were used for data analyses, and Duncan's method was used to compare significant differences between treatments $(p<0.05)$, which are marked with different lowercase letters. All data are the mean \pm standard error of 3 biological replicates with 3 technical replicates. All qRT-PCR validation primers used in the present study are listed in Table 2. 


\section{Declarations}

\section{Ethical approval and consent to participation}

This article does not contain any studies with animals or humans performed by any of the authors. This study complies with institutional, national and international guidelines and legislation.

Consent for publication

Not applicable.

\section{Availability of data and materials}

Not applicable.

\section{Funding}

This work was supported by the National Natural Science Foundation of China (No. 32071866).

\section{Authors' contributions}

$\mathrm{HC}$ and $\mathrm{KH}$ designed and conducted the study. $\mathrm{KH}, \mathrm{CL}, \mathrm{ZZ}, \mathrm{LZ}, \mathrm{CC}$ and $\mathrm{DZ}$ performed the experiments. $\mathrm{HC}$ and $\mathrm{KH}$ drafted the manuscript. All authors contributed to the acquisition of data, interpretation of results and critical discussion and approved the final version of the manuscript.

\section{Declaration of competing interest}

The author declares that this article has not been published, and there are no conflicts of interest.

\section{Acknowledgements}

Not applicable.

\section{References}

1. Windhövel A, Hein I, Dabrowa R, Stockhaus J. Characterization of a novel class of plant homeodomain proteins that bind to the $\mathrm{C} 4$ phosphoenolpyruvate carboxylase gene of Flaveria trinervia. Plant Mol Biol. 2001;45(2):201-214. doi:10.1023/a:1006450005648.

2. Tan QK, Irish VF. The Arabidopsis zinc finger-homeodomain genes encode proteins with unique biochemical properties that are coordinately expressed during floral development. Plant Physiol. 2006;140(3):1095-1108. doi:10.1104/pp.105.070565.

3. Gehring WJ, Affolter M, Bürglin T. Homeodomain proteins. Annu Rev Biochem. 1994;63:487-526. doi:10.1146/annurev.bi.63.070194.002415.

4. Wolberger C. Homeodomain interactions. Curr Opin Struct Biol. 1996;6(1):62-68. doi:10.1016/s0959440x(96)80096-0. 
5. Takatsuji $\mathrm{H}$. Zinc-finger proteins: the classical zinc finger emerges in contemporary plant science. Plant Mol Biol. 1999;39(6):1073-1078. doi:10.1023/a:1006184519697.

6. Hu W, Ma H. Characterization of a novel putative zinc finger gene MIF1: involvement in multiple hormonal regulation of Arabidopsis development. Plant J. 2006;45(3):399-422. doi:10.1111/j.1365313X.2005.02626.x.

7. Hu W, dePamphilis CW, Ma H. Phylogenetic analysis of the plant-specific zinc finger-homeobox and mini zinc finger gene families. J Integr Plant Biol. 2008;50(8):1031-1045. doi:10.1111/j.17447909.2008.00681.x.

8. Wang $H$, Yin $X$, Li X, et al. Genome-wide identification, evolution and expression analysis of the grape (Vitis vinifera L.) zinc finger-homeodomain gene family. Int J Mol Sci. 2014;15(4):5730-5748. Published 2014 Apr 3. doi:10.3390/ijms15045730.

9. Bhattacharjee A, Ghangal R, Garg R, Jain M. Genome-wide analysis of homeobox gene family in legumes: identification, gene duplication and expression profiling. PLoS One. 2015;10(3):e0119198. Published 2015 Mar 6. doi:10.1371/journal.pone.0119198.

10. Khatun K, Nath UK, Robin AHK, et al. Genome-wide analysis and expression profiling of zinc finger homeodomain (ZHD) family genes reveal likely roles in organ development and stress responses in tomato. BMC Genomics. 2017;18(1):695. Published 2017 Sep 6. doi:10.1186/s12864-017-4082-y.

11. Liu M, Sun W, Ma Z, et al. Genome-wide investigation of the AP2/ERF gene family in tartary buckwheat (Fagopyum Tataricum). BMC Plant Biol. 2019;19(1):84. Published 2019 Feb 20. doi:10.1186/s12870-019-1681-6.

12. Liu H, Yang Y, Zhang L. Zinc Finger-Homeodomain Transcriptional Factors (ZF-HDs) in Wheat (Triticum aestivum L.): Identification, Evolution, Expression Analysis and Response to Abiotic Stresses. Plants (Basel). 2021;10(3):593. Published 2021 Mar 22. doi:10.3390/plants10030593.

13. Park HC, Kim ML, Lee SM, et al. Pathogen-induced binding of the soybean zinc finger homeodomain proteins GmZF-HD1 and GmZF-HD2 to two repeats of ATTA homeodomain binding site in the calmodulin isoform 4 (GmCaM4) promoter. Nucleic Acids Res. 2007;35(11):3612-3623. doi:10.1093/nar/gkm273

14. Shalmani A, Muhammad I, Sharif R, et al. Zinc Finger-Homeodomain Genes: Evolution, Functional Differentiation, and Expression Profiling Under Flowering-Related Treatments and Abiotic Stresses in Plants. Evol Bioinform Online. 2019;15:1176934319867930. Published 2019 Sep 5. doi:10.1177/1176934319867930

15. Wang H, Yin X, Li X, et al. Genome-wide identification, evolution and expression analysis of the grape (Vitis vinifera L.) zinc finger-homeodomain gene family. Int J Mol Sci. 2014;15(4):5730-5748. Published 2014 Apr 3. doi:10.3390/ijms15045730.

16. Figueiredo DD, Barros PM, Cordeiro AM, et al. Seven zinc-finger transcription factors are novel regulators of the stress responsive gene OsDREB1B. J Exp Bot. 2012;63(10):3643-3656. doi:10.1093/jxb/ers035. 
17. Mielmann A. The utilisation of lucerne (Medicago sativa ): a review. British Food Journal. 2013; 115.4: 590-600. doi:10.1108/00070701311317865.

18. Chen $\mathrm{H}$, Zeng $\mathrm{Y}$, Yang $\mathrm{Y}$, et al. Allele-aware chromosome-level genome assembly and efficient transgene-free genome editing for the autotetraploid cultivated alfalfa. Nat Commun. 2020;11(1):2494. Published 2020 May 19. doi:10.1038/s41467-020-16338-x.

19. Shen C, Du H, Chen Z, et al. The Chromosome-Level Genome Sequence of the Autotetraploid Alfalfa and Resequencing of Core Germplasms Provide Genomic Resources for Alfalfa Research. Mol Plant. 2020;13(9):1250-1261. doi:10.1016/j.molp.2020.07.003.

20. Liu M, Sun W, Ma Z, et al. Genome-wide investigation of the AP2/ERF gene family in tartary buckwheat (Fagopyum Tataricum). BMC Plant Biol. 2019;19(1):84. Published 2019 Feb 20. doi:10.1186/s12870-019-1681-6.

21. Vimala Y, Lavania S, Lavania UC. Chromosome change and karyotype differentiation-implications in speciation and plant systematics. Nucleus 64, 33-54 (2021). doi:10.1007/s13237-020-00343-y

22. Xu G, Guo C, Shan H, Kong H. Divergence of duplicate genes in exon-intron structure. Proc Natl Acad Sci U S A. 2012;109(4):1187-1192. doi:10.1073/pnas.1109047109.

23. Friedman R, Hughes AL. Gene duplication and the structure of eukaryotic genomes. Genome Res. 2001;11(3):373-381. doi:10.1101/gr.155801.

24. Hu W, Wang $\mathrm{Y}$, Bowers $\mathrm{C}, \mathrm{Ma} \mathrm{H}$. Isolation, sequence analysis, and expression studies of florally expressed cDNAs in Arabidopsis. Plant Mol Biol. 2003;53(4):545-563. doi:10.1023/B:PLAN.0000019063.18097.62.

25. Barth O, Vogt S, Uhlemann R, Zschiesche W, Humbeck K. Stress induced and nuclear localized HIPP26 from Arabidopsis thaliana interacts via its heavy metal associated domain with the drought stress related zinc finger transcription factor ATHB29. Plant Mol Biol. 2009;69(1-2):213-226. doi:10.1007/s11103-008-9419-0.

26. Knight CA, Vogel H, Kroymann J, Shumate A, Witsenboer H, Mitchell-Olds T. Expression profiling and local adaptation of Boechera holboellii populations for water use efficiency across a naturally occurring water stress gradient. Mol Ecol. 2006;15(5):1229-1237. doi:10.1111/j.1365294X.2006.02818.x.

27. Chen $\mathrm{C}$, Chen $\mathrm{H}$, Zhang Y, et al. TBtools: An Integrative Toolkit Developed for Interactive Analyses of Big Biological Data. Mol Plant. 2020;13(8):1194-1202. doi:10.1016/j.molp.2020.06.009.

28. Li H, Handsaker B, Wysoker A, et al. The Sequence Alignment/Map format and SAMtools. Bioinformatics. 2009;25(16):2078-2079. doi:10.1093/bioinformatics/btp352

29. Trapnell C, Roberts A, Goff L, et al. Differential gene and transcript expression analysis of RNA-seq experiments with TopHat and Cufflinks. Nat Protoc. 2012;7(3):562-578. Published 2012 Mar 1. doi:10.1038/nprot.2012.016

30. Gentleman RC, Carey VJ, Bates DM, et al. Bioconductor: open software development for computational biology and bioinformatics. Genome Biol. 2004;5(10):R80. doi:10.1186/gb-2004-5-10r80 


\section{Tables}

Table 1|Related information of MsZF-HD genes 


\section{Gene name Gene locus}

Chromosome location

Length

PI

(aa)

\begin{tabular}{|c|c|c|c|c|c|}
\hline MsMIF-Z1 & MsG0380013130 & chr3:30962255-30962028 & 75 & 8.97 & 8141.2 \\
\hline MsMIF-Z2 & MsG0680030765 & chr6:8778200-8778457 & 85 & 8.88 & 9361.44 \\
\hline MsMIF-Z3 & MsG0880046457 & chr8:73611430-73611660 & 76 & 9.04 & 8305.48 \\
\hline$M s Z H D-Z 1$ & MsG0180004327 & chr1:76449042-76448458 & 194 & 9.16 & 22425.74 \\
\hline MsZHD-Z2 & MsG0580025668 & chr5:22328164-22327376 & 239 & 9.14 & 25973.01 \\
\hline MsZHD-Z3 & MsG0580027925 & chr5:71184035-71184838 & 267 & 6.46 & 29104.05 \\
\hline$M s Z H D-Z 4$ & MsG0680034750 & chr6:93921235-93921759 & 174 & 8.48 & 19211.3 \\
\hline$M s Z H D-Z 5$ & MsG0780036244 & chr7:5192044-5191028 & 338 & 8.2 & 37839.15 \\
\hline MsZHD-Z6 & MsG0880044604 & chr8:45574688-45575857 & 389 & 9.06 & 42303.86 \\
\hline$M s Z H D-Z 7$ & MsG0880045269 & chr8:56721743-56721165 & 192 & 7.08 & 21509.13 \\
\hline$M s Z H D-Z 8$ & MsG0880045282 & chr8:56879882-56880490 & 202 & 7.65 & 22289.51 \\
\hline$M s Z H D-X-1$ & MS.gene020409 & $\begin{array}{l}\text { scaffold51443:180617- } \\
181378\end{array}$ & 253 & 9.12 & 27014.18 \\
\hline MsMIF-X1-1 & MS.gene018941 & chr3.1:27683365-27683093 & 90 & 9.04 & 10019.31 \\
\hline MsMIF-X1-2 & MS.gene061719 & chr8.1:16702902-16702672 & 76 & 9.04 & 8305.48 \\
\hline MsMIF-X2-1 & MS.gene033123 & chr8.2:17333400-17333170 & 76 & 9.04 & 8305.48 \\
\hline MsMIF-X3-1 & MS.gene58432 & chr8.3:15992545-15992775 & 76 & 9.02 & 8265.41 \\
\hline MsMIF-X4-1 & MS.gene044382 & chr8.4:18692730-18692500 & 76 & 9.02 & 8265.41 \\
\hline$M s Z H D-X 1-1$ & MS.gene062215 & chr1.1:57718340-57717756 & 194 & 8.9 & 22419.74 \\
\hline$M s Z H D-X 1-2$ & MS.gene48438 & chr3.1:80006686-80005937 & 249 & 9.12 & 26669.81 \\
\hline$M s Z H D-X 1-3$ & MS.gene047789 & chr5.1:11880941-11881956 & 321 & 9.21 & 36190.73 \\
\hline$M s Z H D-X 1-4$ & MS.gene86236 & chr5.1:23120941-23120186 & 251 & 9.23 & 27137.36 \\
\hline$M s Z H D-X 1-5$ & MS.gene64101 & chr5.1:50377119-50377925 & 268 & 6.46 & 29161.1 \\
\hline$M s Z H D-X 1-6$ & MS.gene000242 & chr6.1:65677061-65677600 & 179 & 8.16 & 19654.82 \\
\hline$M s Z H D-X 1-7$ & MS.gene98674 & chr7.1:31663989-31663597 & 130 & 4.72 & 14239.1 \\
\hline$M s Z H D-X 1-8$ & MS.gene91521 & chr7.1:85201850-85202878 & 342 & 8.2 & 38287.68 \\
\hline$M s Z H D-X 1-9$ & MS.gene012789 & chr8.1:270138-269035 & 367 & 7.56 & 41336.66 \\
\hline
\end{tabular}


$\begin{array}{llllll}M s Z H D-X 1- & \text { MS.gene008887 } & \text { chr8.1:35893129-35892491 } & 212 & 7.65 & 23365.85\end{array}$ $\begin{array}{llllll}\begin{array}{l}\text { MsZHD-X1- } \\ 11\end{array} & \text { MS.gene46290 } & \text { chr8.1:36080136-36080799 } & 233 & 5.81 & 26133.83\end{array}$

\begin{tabular}{lllllll}
\hline $\begin{array}{l}\text { MsZHD-X1- } \\
12\end{array}$ & MS.gene008898 & chr8.1:36089558-36090205 & 247 & 6.18 & 27857.81 \\
\hline $\begin{array}{l}\text { MsZHD-X1- } \\
13\end{array}$ & MS.gene34663 & chr8.1:46373743-46372541 & 400 & 9.06 & 43368.84 \\
\hline
\end{tabular}

\begin{tabular}{|c|c|c|c|c|c|}
\hline$M s Z H D-X 2-1$ & MS.gene88817 & chr1.2:63519845-63521889 & 239 & 8.92 & 27243.13 \\
\hline$M s Z H D-X 2-2$ & MS.gene07447 & chr1.2:63618407-63617808 & 199 & 9.14 & 22835.08 \\
\hline$M s Z H D-X 2-3$ & MS.gene53939 & chr3.2:1099072-1098620 & 150 & 5.09 & 16946.49 \\
\hline$M s Z H D-X 2-4$ & MS.gene052178 & chr3.2:83875635-83874811 & 274 & 6.08 & 29683.68 \\
\hline$M s Z H D-X 2-5$ & MS.gene010515 & chr5.2:11751825-11752855 & 326 & 9.21 & 36726.33 \\
\hline$M s Z H D-X 2-6$ & MS.gene027744 & chr5.2:22593459-22592704 & 251 & 9.23 & 27137.36 \\
\hline$M s Z H D-X 2-7$ & MS.gene010125 & chr5.2:55657198-55657992 & 264 & 6.76 & 28717.69 \\
\hline$M s Z H D-X 2-8$ & MS.gene043241 & chr7.2:87132177-87133202 & 341 & 8.2 & 38213.59 \\
\hline$M s Z H D-X 2-9$ & MS.gene95053 & chr8.2:261015 -259891 & 374 & 7.32 & 42099.57 \\
\hline $\begin{array}{l}\text { MsZZHD-X2- } \\
10\end{array}$ & MS.gene056421 & chr8.2:33683282-33682785 & 165 & 5.15 & 17608.69 \\
\hline
\end{tabular}

$\begin{array}{llllll}\text { MsZHD-X2- } & \text { MS.gene056417 } & \text { chr8.2:33735099-33734401 } & 232 & 8.1 & 25773.17\end{array}$

\begin{tabular}{lllllll}
\hline $\begin{array}{l}\text { MsZHD-X2- } \\
12\end{array}$ & MS.gene35938 & chr8.2:41933556-41932375 & 393 & 9.06 & 42675.27 \\
\hline $\begin{array}{l}\text { MsZHD-X2- } \\
13\end{array}$ & MS.gene035434 & chr8.2:51873360-51872190 & 151 & 6.65 & 17317.73 \\
\hline
\end{tabular}

\begin{tabular}{llllll}
\hline $\begin{array}{l}\text { MsZHD-X2- } \\
14\end{array}$ & MS.gene035426 & chr8.2:51974569-51974243 & 108 & 5.46 & 11973.5
\end{tabular}

\begin{tabular}{lllllll}
\hline MsZHD-X3-1 & MS.gene013045 & chr3.3:83536735-83535986 & 249 & 9.12 & 26669.81 \\
\hline MsZHD-X3-2 & MS.gene02448 & chr5.3:11832602-11833168 & 188 & 9.3 & 21537.37 \\
\hline MsZHD-X3-3 & MS.gene29146 & chr5.3:21737080-21736325 & 251 & 9.23 & 27137.36 \\
\hline MsZHD-X3-4 & MS.gene061947 & chr5.3:51674272-51675075 & 267 & 6.46 & 29104.05 \\
\hline MsZHD-X3-5 & MS.gene79601 & chr7.3:87786119-87787153 & 344 & 8.2 & 38534.88 \\
\hline MsZHD-X3-6 & MS.gene042050 & chr8.3:206351-205122 & 306 & 8.21 & 34618.71 \\
\hline MsZHD-X3-7 & MS.gene067921 & chr8.3:33702409-33701960 & 149 & 5.54 & 16220.24 \\
\hline
\end{tabular}




\begin{tabular}{|lllllll} 
MsZHD-X3-8 & MS.gene067910 & chr8.3:33916426-33917166 & 246 & 6.31 & 27736.74 \\
\hline MsZHD-X3-9 & MS.gene045953 & chr8.3:43574252-43573254 & 332 & 8.86 & 36169.03 \\
\hline MsZHD-X4-1 & MS.gene004926 & chr1.4:63200375-63199791 & 194 & 8.91 & 22386.59 \\
\hline MsZHD-X4-2 & MS.gene59378 & chr5.4:12454799-12455817 & 322 & 9.21 & 36291.88 \\
\hline MsZHD-X4-3 & MS.gene44274 & chr5.4:22839634-22838879 & 251 & 9.23 & 27137.36 \\
\hline MsZHD-X4-4 & MS.gene050020 & chr5.4:51440438-51441241 & 267 & 6.46 & 29150.13 \\
\hline MsZHD-X4-5 & MS.gene021696 & chr7.4:88978290-88978985 & 341 & 8.2 & 38163.53 \\
\hline MsZHD-X4-6 & MS.gene43476 & chr8.4:277389-276562 & 275 & 8.69 & 31307.94 \\
\hline MsZHD-X4-7 & MS.gene048325 & chr8.4:34663911-34664555 & 214 & 6.45 & 23864.96
\end{tabular}

Table 2| List of qRT-PCR validation primers used in the present study.

\begin{tabular}{llr} 
Gene ID & Forward primer $\left(5^{\prime}-3^{\prime}\right)$ & Reverse primer $\left(5^{\prime}-3^{\prime}\right)$ \\
\hline MsZHD-X1-6 & CGGTAAACAGCCTCCAATG & CCTAACTTCTCAGCAAATCCC \\
\hline MsZHD-X1-8 & AACACTTTAGCCAAACGAGA & GATGAACCATTAGCACGAAC \\
\hline MsZHD-X1-11 & GGAGGCTACGCTCTTGATG & AGTCGGCTGAGGGAAACG \\
\hline MsZHD-X2-12 & TCCCTCAAATGTGCTGCTT & GTGGTGGTTGTGGTGGTG \\
\hline MsZHD-X3-4 & GACGGTTGTTGCGAGTTTAT & CAACGGTCTGTGATGTGCC \\
\hline MsZHD-X4-3 & TTGATGGTTGCGGTGAGT & GTGGTGGATGGTGATGATGT \\
\hline MsZHD-Z2 & TTGATGGTTGCGGTGAGT & GTGGTGGATGGTGATGATGT \\
\hline MsZHD-Z3 & GACGGTTGTTGCGAGTTTAT & CAACGGTCTGTGATGTGCC \\
\hline MsZHD-Z5 & AACACTTTAGCCAAACGAGA & GATGAACCATTAGCACGAAC
\end{tabular}

\section{Figures}




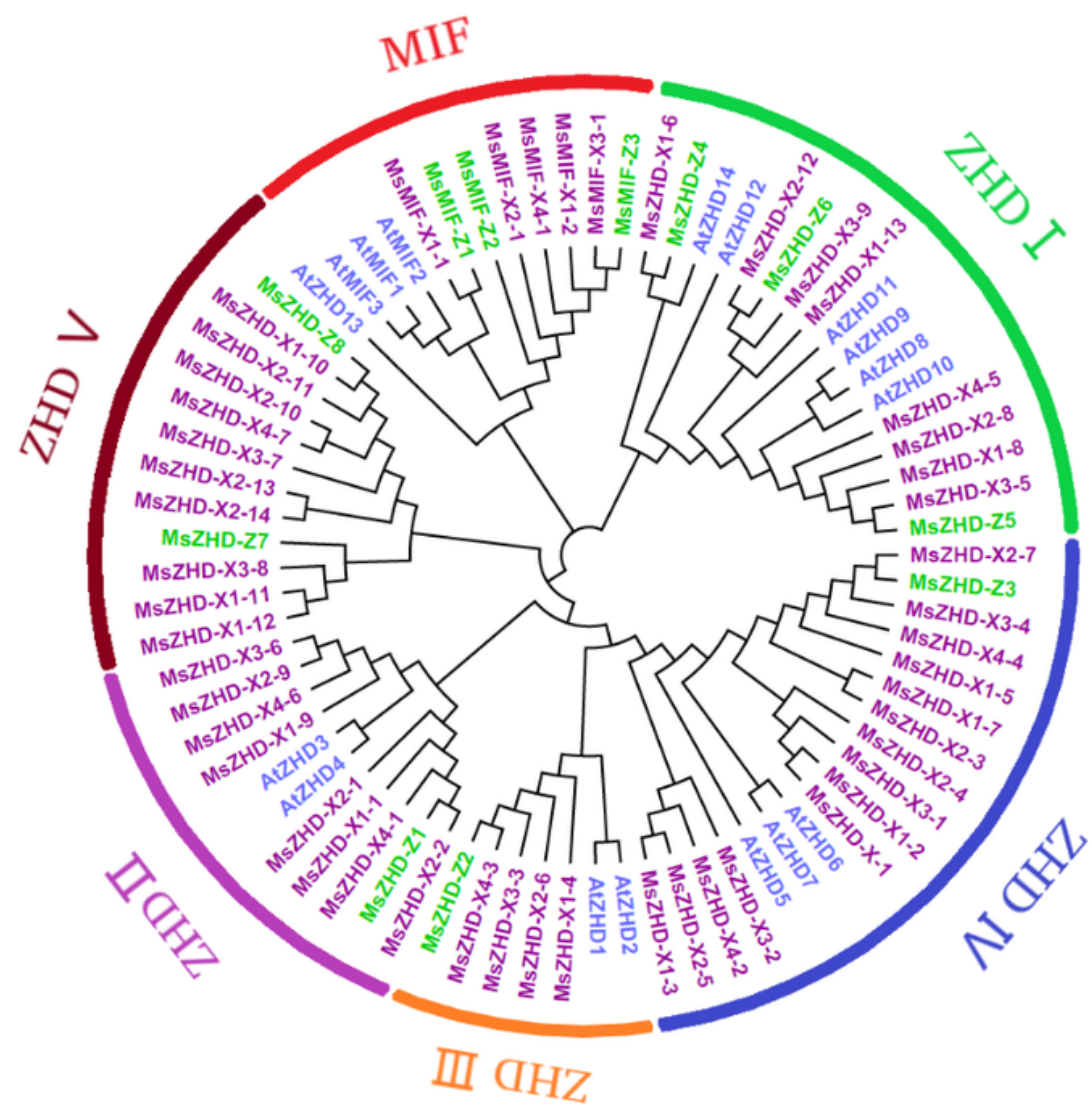

Figure 1

Unrooted phylogenetic tree based on the relationship between Medicago sativa L. and Arabidopsis thaliana. Coloured branches indicate different subfamilies. 


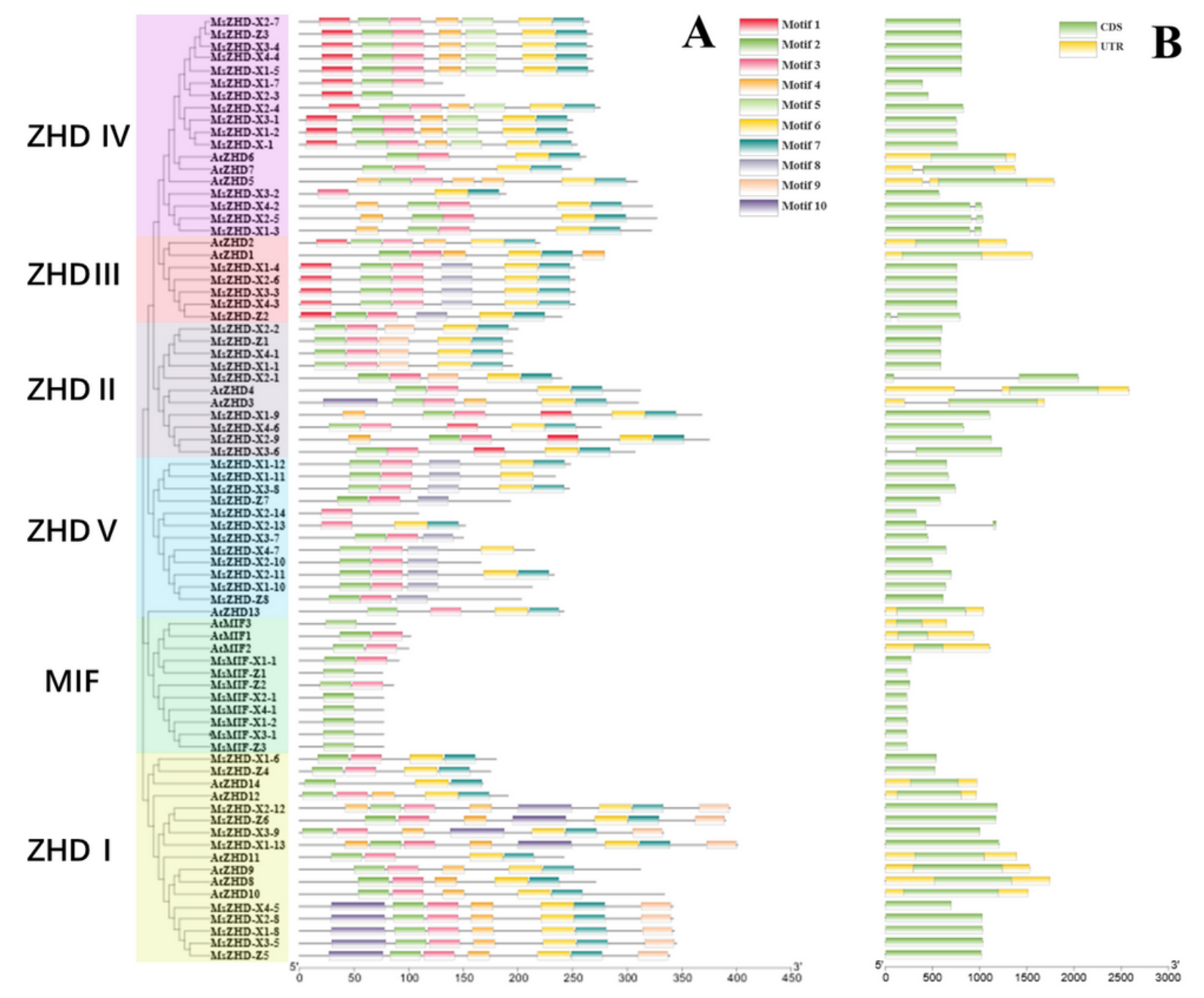

Figure 2

Phylogenetic relationships, gene structures and architectures of the conserved protein motifs. (A) The motif composition of the MsZF-HD proteins. The motifs, numbered 1-10, are displayed in different colored boxes. (B) Exon-intron structures of the MsZF-HD genes. Yellow boxes indicate untranslated 5'and 3'-regions; green boxes indicate exons; and black lines indicate introns. 


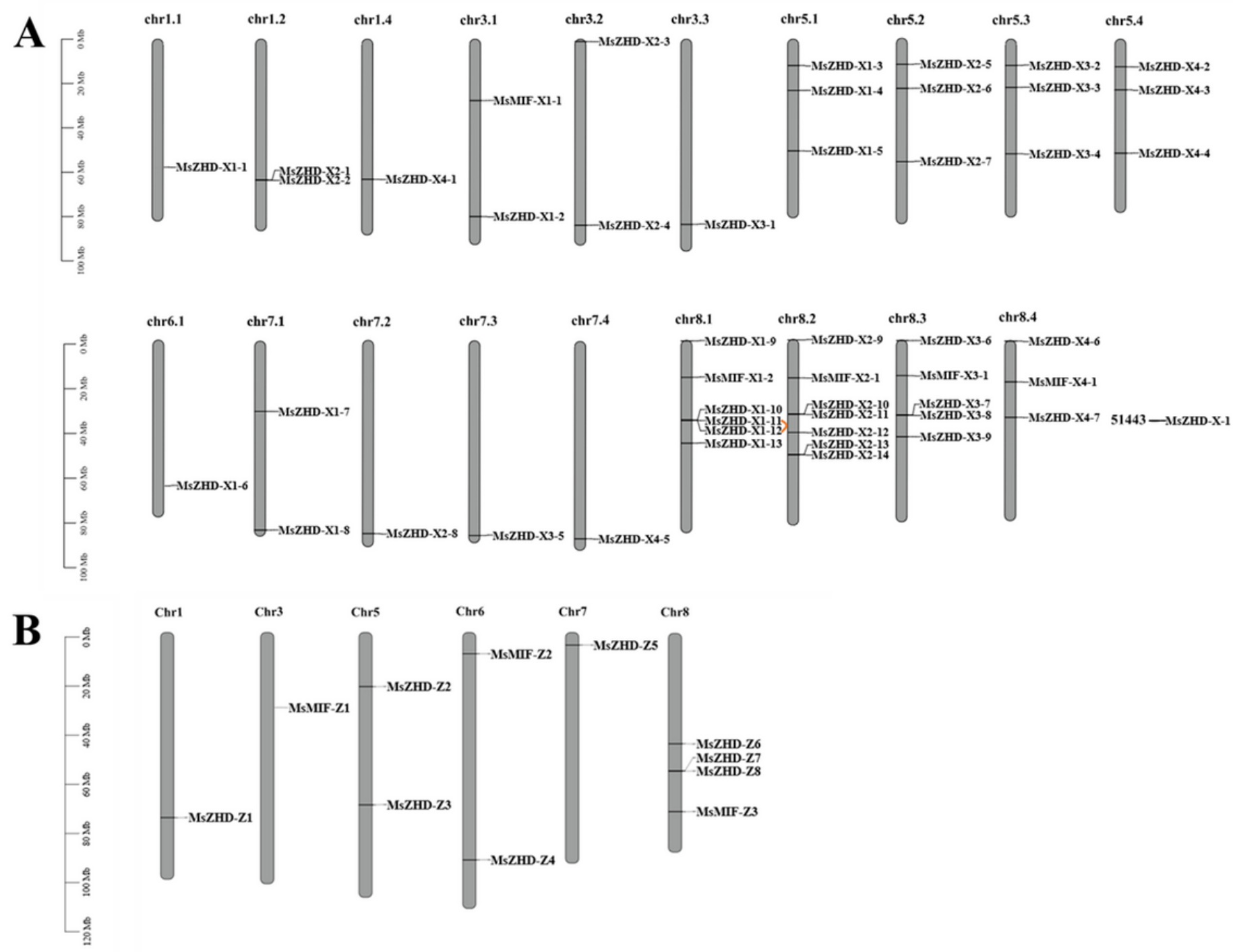

\section{Figure 3}

Chromosomal location of Medicago sativa L. (A) XinJiangDa Ye. (B) Zhongmu No. 1. 


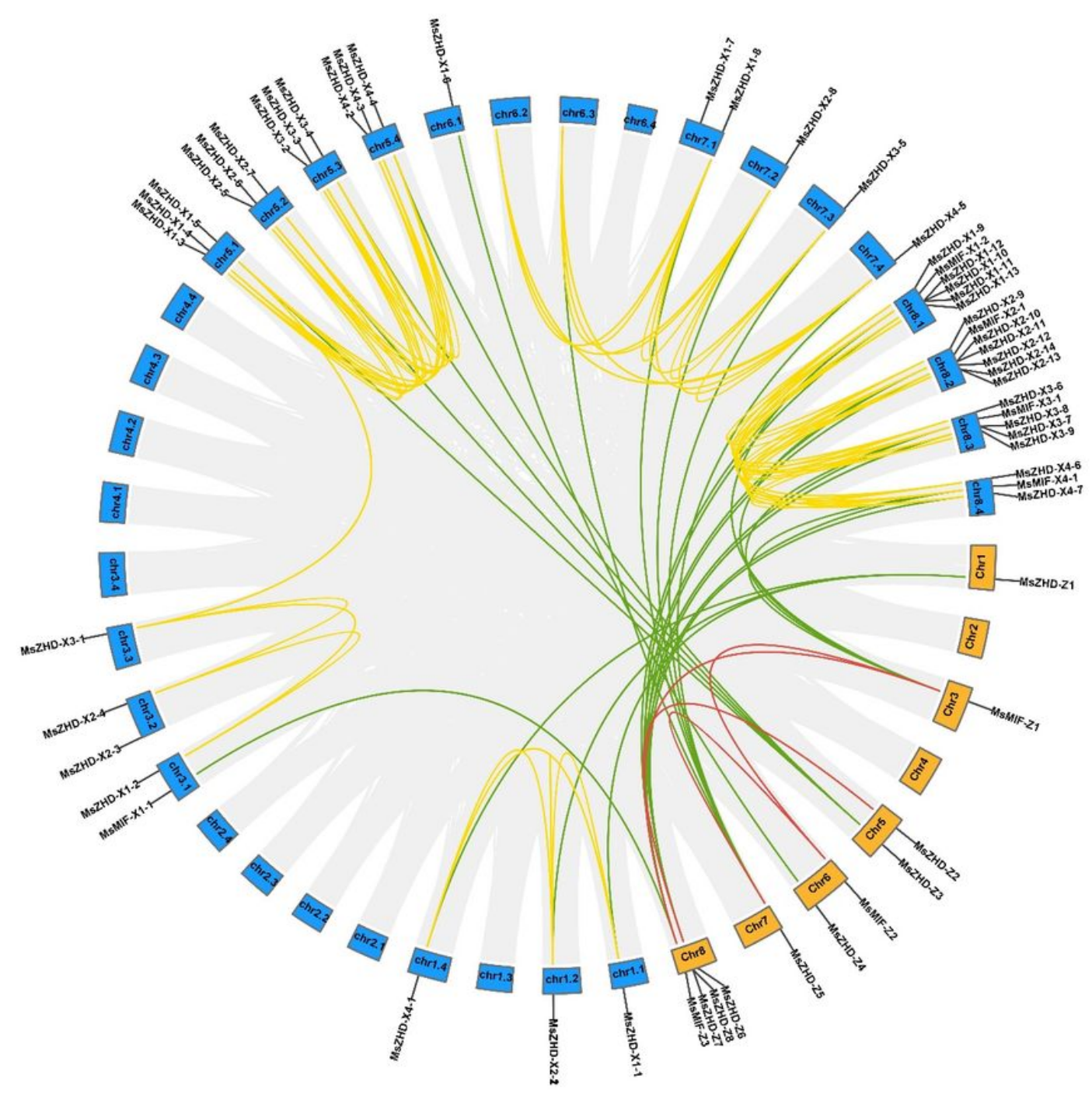

\section{Figure 4}

Orthologous genes of MsZF-HD genes between XinJiangDa Ye and Zhongmu No. 1. Gray lines in the background indicate the collinear blocks within two different varieties' genomes; red and yellow lines highlight the syntenic MsZF-HD gene pairs in XinJiangDa Ye and Zhongmu No. 1 respectively; green lines highlight the syntenic MsZF-HD gene pairs between the two different varieties. 

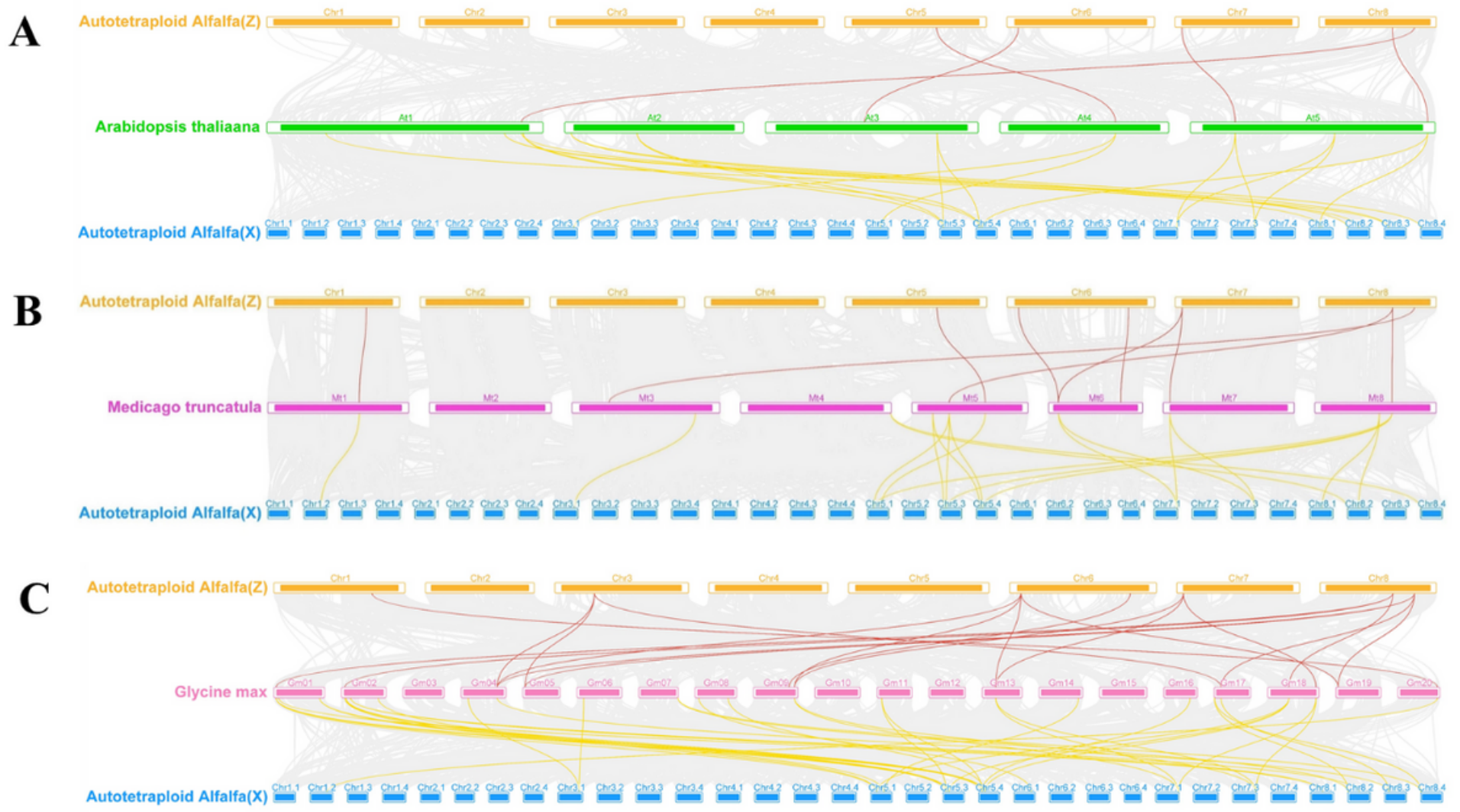

\section{Figure 5}

Orthologous genes of ZF-HD genes between Medicago sativa L, Arabidopsis thaliana, Medicago truncatula and Glycine max. Gray lines in the background indicate the collinear blocks within alfalfa and other plant genomes; red and yellow lines highlight the syntenic MsZF-HD gene pairs of Xin JiangDa Ye and Zhongmu No. 1 respectively. 


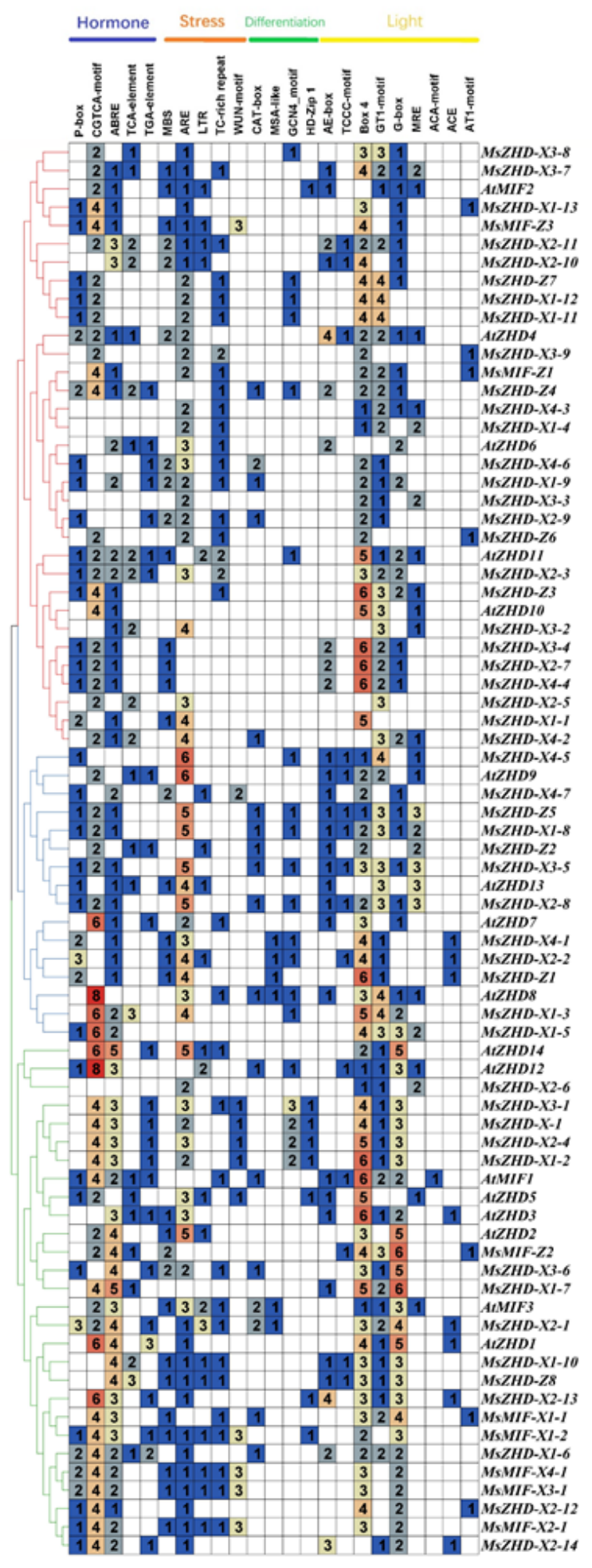

Figure 6

Cis-elements in the promoter region of the MsZF-HD genes. The number of cis-elements in the promoter region of each MsZF-HD genes ( $2 \mathrm{~kb}$ upstream of the translation start site) is indicated by the different colors and numbers in the grid. 
A
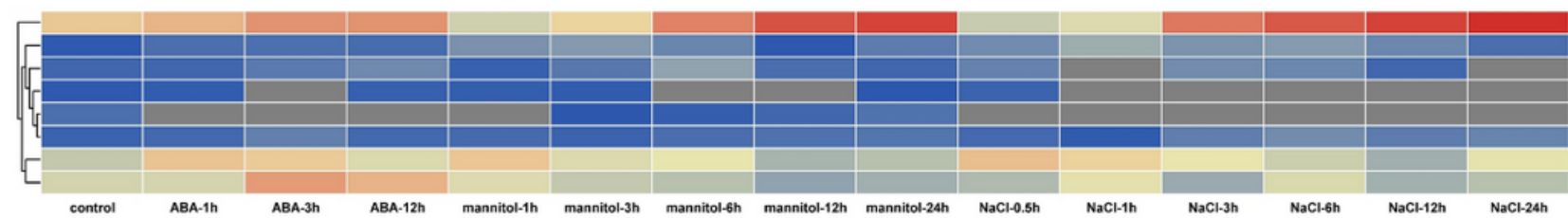

B

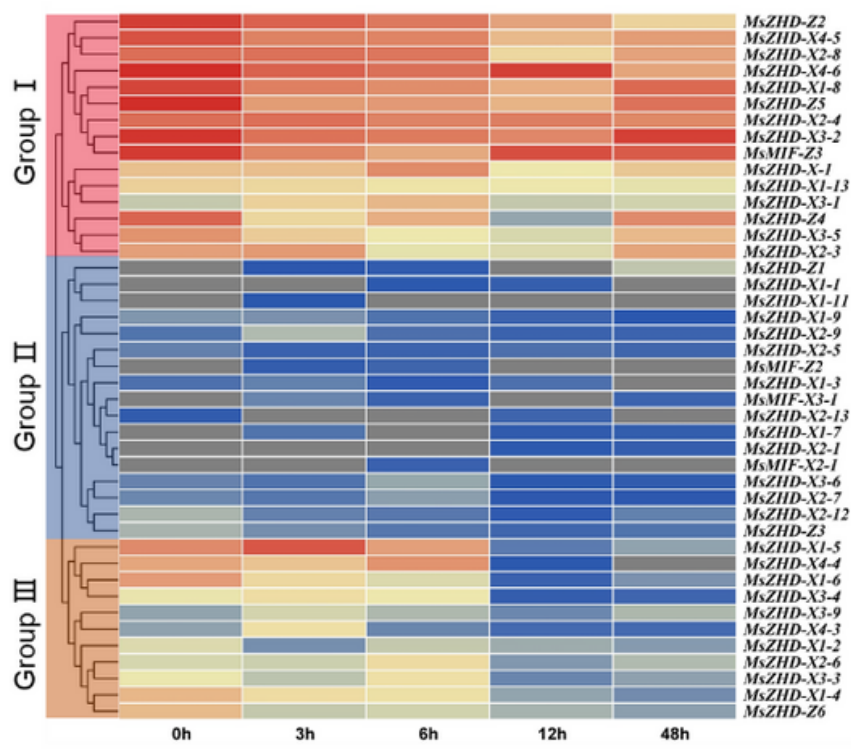

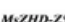

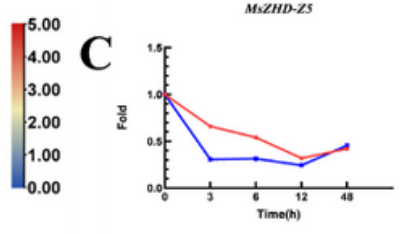

M.ZHD-X3-4
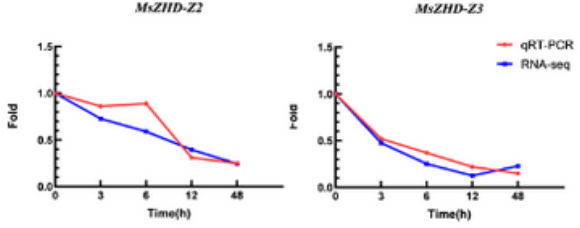

$M \times z H D-X+3$
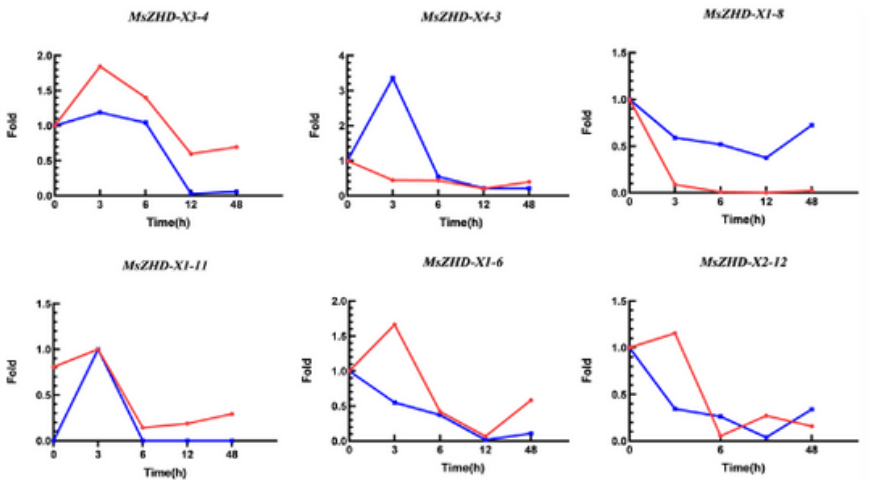

\section{Figure 7}

Expression of MsZF-HD genes in response to abiotic treatment. (A) The express patern of MsZF-HD genes under different abiotic treatment ( $250 \mathrm{mM} \mathrm{NaCl}, 10 \mathrm{M} \mathrm{ABA}$ and $400 \mathrm{mM}$ mannitol). The FPKMs were calculated for expression values from RNA-Seq data. (B) The MsZF-HD genes were clustered into three groups basing on expressional profiles under alkaline stress. (C) qRT-PCR verification of the RNAseq result.

\section{Supplementary Files}

This is a list of supplementary files associated with this preprint. Click to download.

- Aditionalfile.xlsx 